\title{
Posttreatment Infarct Volumes when Compared with 24-Hour and 90-Day Clinical Outcomes: Insights from the REVASCAT Randomized Controlled Trial
}

\author{
(D)F.S. Al-Ajlan, (D) A.S. Al Sultan, DP. Minhas, Dz. Assis, (D) M.A. de Miquel, (D) M. Millán, (DL. San Román, (D) A. Tomassello, \\ (D)A.M. Demchuk, DT.G. Jovin, (DP. Cuadras, (D) A. Dávalos, (D). Goyal, and DB.K. Menon; for the REVASCAT Investigators
}

\begin{abstract}
BACKGROUND AND PURPOSE: Endovascular therapy has become the standard of care for patients with disabling anterior circulation ischemic stroke due to proximal intracranial thrombi. Our aim was to determine whether the beneficial effect of endovascular treatment on functional outcome could be explained by a reduction in posttreatment infarct volume in the Endovascular Revascularization With Solitaire Device Versus Best Medical Therapy in Anterior Circulation Stroke Within 8 Hours (REVASCAT) trial.
\end{abstract}

MATERIALS AND METHODS: The REVASCAT trial was a multicenter randomized open-label trial with blinded outcome evaluation. Among 206 enrolled subjects (endovascular treatment, $n=103$; control, $n=103$ ), posttreatment infarct volume was measured in 204 subjects. Posttreatment infarct volumes were compared with treatment assignment and recanalization status. Appropriate statistical models were used to assess the relationship among baseline clinical and imaging variables, posttreatment infarct volume, the 24-hour NIHSS score, and functional status with the 90-day modified Rankin Scale score.

RESULTS: The median posttreatment infarct volume in all subjects was $23.7 \mathrm{~mL}$ (interquartile range $=68.9 \mathrm{~mL}$ ) and $16.3 \mathrm{~mL}$ (interquartile range $=50.2 \mathrm{~mL}$ ) in the endovascular treatment arm and $38.6 \mathrm{~mL}$ (interquartile range $=74.9 \mathrm{~mL}$ ) in the control arm $(P=.02 \mathrm{for}$ endovascular treatment versus control subjects). Baseline NIHSS $(P<.01)$, site of occlusion $(P<.03)$, baseline NCCT ASPECTS $(P<.01)$, and recanalization status $(P=.02)$ were independently associated with posttreatment infarct volume. Baseline NIHSS $(P<.01)$, time from symptom onset to randomization $(P=.02)$, treatment type $(P=.04)$, and recanalization status $(P<.01)$ were independently associated with the 24-hour NIHSS scores. The 24-hour NIHSS score strongly mediated the relationship between treatment type and 90 -day mRS $(P<.01$ for indirect effect when adjusted for age), while posttreatment infarct volume did not $(P=.26)$.

CONCLUSIONS: Endovascular treatment saves brain and improves 90 -day clinical outcomes primarily through a beneficial effect on the 24-hour stroke severity.

ABBREVIATIONS: $\mathrm{EVT}=$ endovascular treatment; $\mathrm{IQR}=$ interquartile range

n 2015, five randomized clinical trials revolutionized stroke care by confirming the benefit of modern endovascular therapy

\footnotetext{
Received June 11, 2017; accepted after revision August 16.

From the Department of Clinical Neurosciences and Department of Radiology (F.S.A.-A., A.S.A.S., P.M., Z.A., A.M.D., M.G., B.K.M.), Cumming School of Medicine, University of Calgary, Calgary, Alberta, Canada; Department of Neurosciences at King Faisal Specialist Hospital and Research Centre (F.S.A.-A.), Riyadh, Saudi Arabia; Stroke Unit (M.A.d.M.), Neurology Department, Hospital de Bellvitge, L'Hospitalet de Llobregat, Barcelona, Spain; Stroke Unit (M.M., P.C., A.D.), Department of Neurosciences and Department of Radiology, Hospital Germans Trias, Universitat Autosome de Barcelona, Badalona (Barcelona), Spain; Stroke Unit (L.S.R.), Neurology Department, Hospital Clínic, Barcelona, Spain; Stroke Unit (A.T.), Neurology Department, Hospital Vall d'Hebron, Barcelona, Spain; and Stroke Institute (T.G.J.), Department of Neurology, University of Pittsburgh Medical Center, Pittsburgh, Pennsylvania.

REVASCAT was funded by a local independent Catalan institution (Fundació Ictus Malaltia Vascular) through an unrestricted grant from the manufacturer of the device (Covidien). This project has been partially supported by a grant from the Spanish Ministry of Health cofinanced by FEDER (Instituto de Salud Carlos III, RETICS-INVICTUS PLUS, RD 16/0019).
}

(EVT) in patients with acute ischemic stroke due to proximal anterior circulation occlusion. ${ }^{1-5}$ Fast reperfusion played an important role in the success of these trials, likely by saving brain that would otherwise have infarcted without reperfusion.

Although posttreatment infarct volumes are routinely used in experimental models of focal cerebral ischemia, ${ }^{6}$ human clinical trials have preferred to use ordinal scales like the modified Rankin Scale, the National Institutes of Health Stroke Scale, or the Barthel Index to primarily measure posttreatment outcomes. ${ }^{7-9}$ This preference is, in part, because past human studies attempting to correlate posttreatment infarct volumes to clinical outcomes have

Clinical Trial Registration: https://clinicaltrials.gov/ct2/show/NCT01692379.

Please address correspondence to Bijoy K. Menon, MD, MSc, Foothills Medical Centre, 1403 29th St NW, Calgary, AB T2N 2T9 Canada; e-mail: docbijoymenon@gmail.com; @bijoymenon

- Indicates open access to non-subscribers at www.ajnr.org

http://dx.doi.org/10.3174/ajnr.A5463 
yielded contradictory results. ${ }^{10,11-13}$ On the other hand, successful recanalization has been shown to be strongly associated with improved functional outcomes and reduced mortality. ${ }^{14}$ Data from the recent Endovascular Treatment for Small Core and Proximal Occlusion Ischemic Stroke (ESCAPE) trial showed that the successful recanalization and reduction in posttreatment infarct volume were associated with better clinical outcome. ${ }^{15}$ The purpose of this analysis was to analyze the relationship among baseline clinical characteristics, type of treatment, recanalization status, posttreatment infarct volumes, and the 24-hour and 90-day clinical outcomes from the Endovascular Revascularization With Solitaire Device Versus Best Medical Therapy in Anterior Circulation Stroke Within 8 Hours (REVASCAT) trial. ${ }^{5}$

\section{MATERIALS AND METHODS}

Methodologic details of the REVASCAT trial have been reported elsewhere. ${ }^{5}$ Briefly, the REVASCAT trial was a prospective, multicenter, randomized, controlled, open, blinded-end point trial. Patients were randomized 1:1 to mechanical embolectomy with the stent retriever Solitaire FR (Covidien, Irvine, California) versus best medical management alone. Eligible patients were between 18 and 80 years of age, had an occlusion in the proximal anterior circulation and could be treated within 8 hours of symptom onset, and had a prestroke modified Rankin Scale score of $\leq 1$ and a baseline score of at least 6 points on the National Institutes of Health Stroke Scale score. The main exclusion criteria on imaging were evidence of a large ischemic core, as indicated by an Alberta Stroke Program Early CT Score of $<7$ on CT or a score of $<6$ on diffusion-weighted MR imaging. All patients or their surrogates provided written informed consent. The primary outcome was disability at 90 days on the mRS. ${ }^{16}$ Secondary outcomes included centrally adjudicated infarct volumes on CT or MR imaging at 24-36 hours and vessel recanalization on CT angiography or MR angiography at 24-36 hours.

\section{Imaging}

The trial protocol included follow-up MR imaging with MR angiography or noncontrast CT with CT angiography at 24-36 hours from stroke-symptom onset.

Diffusion-weighted imaging was the technique of choice for measurement of posttreatment infarct volume. If DWI was not available, an NCCT scan was chosen for measurement. Two readers (F.S.A.-A. and P.M.) used Quantomo (Cybertrial, Calgary, Alberta, Canada) ${ }^{17}$ to delineate infarct and measure posttreatment infarct volumes (in milliliters) while being blinded to all other clinical and imaging information. Manual adjustments to delineate infarct boundaries were performed when necessary. If the infarct showed hemorrhagic conversion, the hemorrhage regions were incorporated within the boundaries of the infarct. Posttreatment infarct volume was measured with NCCT in 189 subjects and with MR imaging in 15 subjects. Successful recanalization was defined with the modified Thrombolysis in Cerebral Infarction score ( $2 \mathrm{~b}$ or 3 ) on conventional angiography in the intervention arm and the modified arterial occlusive lesion core ( 2 or 3 ) on 24-hour CTA or MRA among subjects in the control arm. $^{18}$

\section{Statistical Analysis}

Because posttreatment infarct volume did not have a normal distribution, the Wilcoxon rank sum test was used to test for differences in volumes between treatment and control subjects and between subjects achieving and not achieving recanalization.

Generalized linear regression was used to model the association between treatment and posttreatment infarct volume, adjusting for prespecified variables (age, sex, baseline NIHSS, baseline site of occlusion [internal carotid artery versus M1 or M2 of the middle cerebral artery], intravenous alteplase [yes versus no], and time from stroke onset to randomization). A cube root transformation of posttreatment infarct volume best satisfied the assumptions of this model (normality of residuals and homoscedasticity) and was used for this analysis. A similar model was used to model the association between treatment and the 24-hour NIHSS score.

A generalized linear model with log-link was used to report rate/risk ratios for functional independence ( $\mathrm{mRS} 0-2$ at 90 days) and 90-day mortality in the highest-versus-lowest posttreatment infarct volume quartile. Generalized linear regression with backward elimination was used to arrive at a final parsimonious model that reports the association between treatment type and 90-day $\mathrm{mRS}$ after adjusting for the 24-hour NIHSS score and posttreatment infarct volume. The template of Baron and Kenny ${ }^{19}$ was used to perform mediational analysis (http://www.ats.ucla.edu/ stat/stata/faq/mulmediation.htm), testing whether the 24-hour NIHSS score and posttreatment infarct volume (potential mediating variables) mediate partially or completely (indirect effect) the association between treatment type and 90-day mRS. All 3 causal steps necessary for mediation analysis as proposed by Baron and Kenny were satisfied (Fig 1). All statistical analyses were performed in STATA/MP version 14.0 (StataCorp, College Station, Texas). Statistical significance was assessed at $\alpha<.05$ in all analyses.

\section{RESULTS}

A total of 206 patients (103 in the EVT arm versus 103 in the control arm) were enrolled in the study. Median baseline NCCT ASPECTS was 7 in the EVT group and 8 in the control group. Intravenous alteplase was administered to $68.0 \%$ of patients in the EVT group and $77.7 \%$ of those in the control group. The median time from stroke onset to randomization was 225 minutes.

Median posttreatment infarct volume in all study participants was $23.7 \mathrm{~mL}$ (interquartile range $[\mathrm{IQR}]=68.9 \mathrm{~mL}$ ) and $16.3 \mathrm{~mL}$ $(\mathrm{IQR}=50.2 \mathrm{~mL})$ in the EVT arm and $38.6 \mathrm{~mL}(\mathrm{IQR}=74.9 \mathrm{~mL})$ in the control arm $(P=.02)$. Median posttreatment infarct volume in the EVT arm in recanalizers was $14.6 \mathrm{~mL}(\mathrm{IQR}=7.8-46.2$ $\mathrm{mL})$ versus $92.9 \mathrm{~mL}(\mathrm{IQR}=14.6-233.1 \mathrm{~mL})$ in the nonrecanalizers $(P=.05)$. The median posttreatment infarct volume in the control arm in recanalizers was $18.1 \mathrm{~mL}(\mathrm{IQR}=8.4-76.7 \mathrm{~mL}$ ) versus $52.8 \mathrm{~mL}(\mathrm{IQR}=18.5-109.5 \mathrm{~mL})$ in the nonrecanalizers $(P=.02)$.

Baseline NIHSS score $(P<.01)$, site of occlusion $(P<.03)$, baseline NCCT ASPECTS $(P<.01)$, and recanalization status $(P=.02)$ were independently associated with posttreatment infarct volume (cube root-transformed), while age, sex, treatment type, intravenous alteplase, and time from symptom onset to randomization were not $(P>.05)$. The baseline NIHSS score $(P<$ 
pared with patients in the lowest quartile (risk ratio $=3.88 ; 95 \%$ CI, 1.39-10.77).

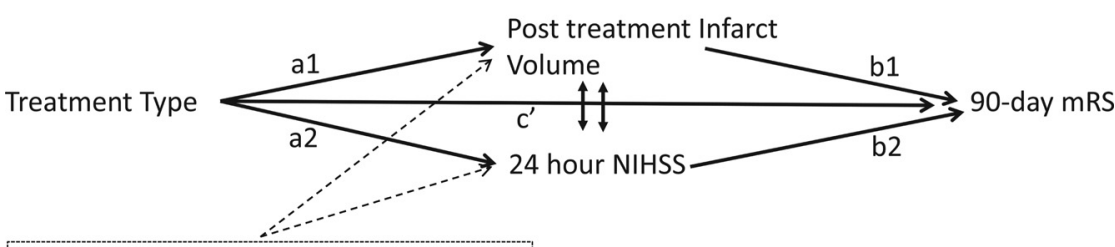
In a parsimonious model obtained after including all baseline clinical and imaging variables, treatment type, and recanalization status with 90-day $\mathrm{mRS}$ as outcome, treatment type was not independently associated with 90-day mRS

FIG 1. A pictorial representation of the causal pathway in patients with acute ischemic stroke. Treatment type (EVT or control) determines posttreatment infarct volume and the 24-hour NIHSS score along with other variables such as age, sex, baseline clinical and imaging markers of stroke severity, time to treatment, and recanalization status (independent variables). Posttreatment infarct volume and the 24-hour NIHSS score (mediator variables) likely then determine 90-day $\mathrm{mRS}$ (dependent variable). The total effect of the independent variables on the dependent variables consists of a direct effect $c^{\prime}$ (as shown in the image) and indirect effects. The indirect or mediating effect of each mediator variable on the association between independent variables and dependent variables is determined by computing the product of the regression coefficients al $\times$ bl or a2 $\times$ b2 as appropriate. In our study, the 2 mediator variables (posttreatment infarct volume and the 24-hour NIHSS score) are significantly correlated. Analysis suggests that the age-adjusted 24-hour NIHSS score mediates strongly the relationship between treatment type and 90-day mRS ( $P<.01$ for indirect effect when adjusted for age), while posttreatment infarct volume does not $(P=.26)$.

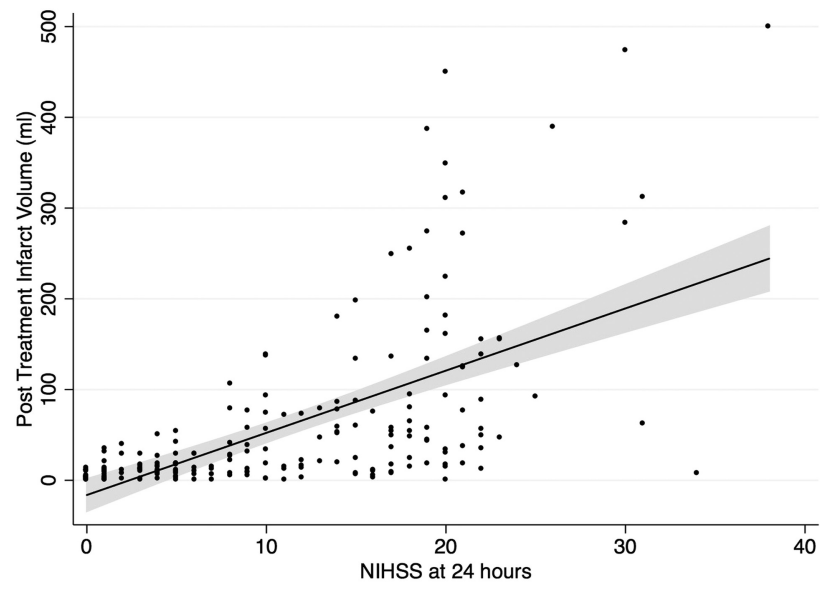

FIG 2. Relationship between the 24-hour NIHSS score and posttreatment infarct volume in milliliters.

$.01)$, time from symptom onset to randomization $(P=.02)$, treatment type $(P=.04)$, and recanalization status $(P<.01)$ were independently associated with the 24-hour NIHSS score, while age, sex, site of occlusion, baseline NCCT ASPECTS, and intravenous alteplase were not $(P>.05)$. A significant correlation was seen between posttreatment infarct volume and the 24-hour NIHSS score $(P<.01$, Fig 2$)$.

Distribution of posttreatment infarct volume versus 90-day $\mathrm{mRS}$ is shown in Fig 3. Patients in the lowest quartile for posttreatment infarct volume were 4 times more likely to achieve functional independence at 90 days (mRS 0-2) compared with patients in the highest quartile (rate ratio $=3.92 ; 95 \% \mathrm{CI}, 0.66$ 23.16). Patients in the highest quartile of posttreatment infarct volume were 4 times more likely to die at 90 days (mRS 6) com- significantly smaller posttreatment infarct volumes only when recanalization was achieved. Subjects in the control arm also achieved smaller posttreatment infarct volumes with recanalization. Our results are therefore consistent with the analysis from the ESCAPE trial that showed similar findings. ${ }^{15}$ We also found that NIHSS measured at 24 hours, though significantly correlated with posttreatment infarct volume, is a better determinant of 90-day mRS than the posttreatment infarct volume.

The causal chain in acute ischemic stroke that explains 90-day clinical outcome is complex. Baseline clinical variables such as age and baseline stroke severity as measured clinically (NIHSS) or on imaging (NCCT ASPECTS and site of occlusion) are known prognostic determinants. Early recanalization as a result of treatment (EVT, intravenous alteplase) also affects 90-day clinical outcomes. Outcomes measured earlier such as posttreatment infarct volume or the 24-hour NIHSS score may only be used as surrogates for 90-day clinical outcome if they capture the effect of these baseline clinical, imaging, and treatment variables on 90-day clinical outcome almost entirely. Our analysis shows that the age-adjusted 24-hour NIHSS score strongly mediates the relationship between treatment type and 90-day outcome and that posttreatment infarct volume does not contribute any further to mediating this relationship. Although we found a robust relationship between the 24-hour NIHSS score and posttreatment infarct volume (Fig 2), the effects of brain eloquence and other prognostic determinants such as diabetes, hypertension, or other medical comorbidities are more likely to be captured by a clinical measure such as the NIHSS compared with an imaging measure like posttreatment infarct volume. Of note, recent analysis from the ESCAPE trial showed that analysis of NIHSS trajectories from baseline to day 5 may be better than the 24-hour NIHSS score alone in determining 90-day mRS. ${ }^{20}$ 


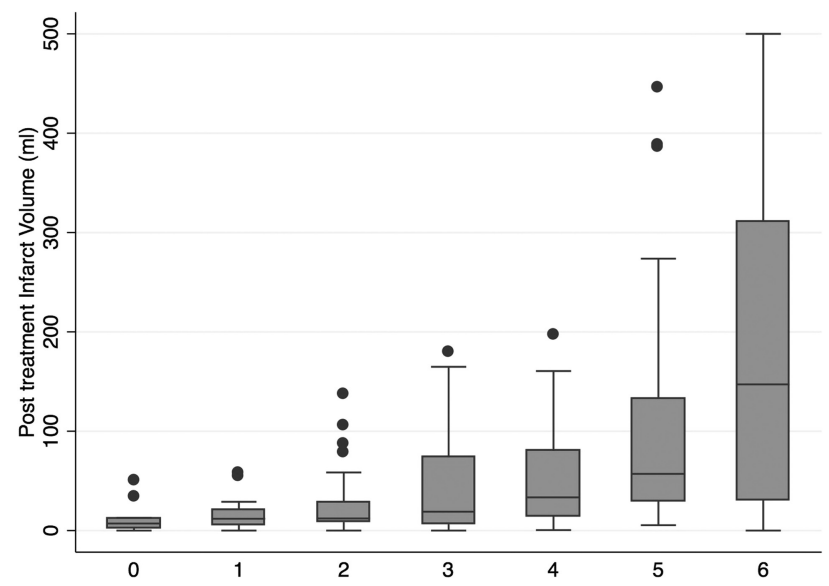

FIG 3. Relationship between 90 -day mRS and posttreatment infarct volume in milliliters.

Aging is a complex medical phenomenon having effects on health beyond direct effects exerted through increasing prevalence of known diseases. ${ }^{21}$ Aging is an important determinant of clinical outcomes in patients with acute ischemic stroke. Our analysis shows that age influences 90-day clinical outcome that is independent of posttreatment infarct volume and the 24-hour NIHSS score. Our analysis therefore emphasizes that surrogate outcomes in stroke such as the 24-hour NIHSS score or even posttreatment infarct volume will lose precision if patient age is not considered.

Our study has limitations. We could not do a complete exploration of the effect of all independent and mediating variables on 90-day clinical outcomes due to the limited sample size. Posttreatment infarct volumes were measured using 24- to 48-hour MR imaging or CT. MR imaging may be more sensitive than CT, and measuring infarct volumes late (even at 30 days) may offer more precise estimates of dead tissue than at 24-48 hours. Finally, although the 24-hour NIHSS score captures brain eloquence, we did not use imaging measures to better delineate eloquence.

\section{CONCLUSIONS}

Our results support primary findings from the REVASCAT trial that endovascular treatment saves brain and improves 24-hour (imaging and clinical outcomes) and 90-day clinical outcomes. The effect of EVT on 90-day clinical outcome is primarily mediated through an effect on the 24-hour NIHSS score.

Disclosures: Maria A. de Miquel—UNRELATED: Board Membership: Fundació Ictus. Andrew M. Demchuk—RELATED: Consulting Fee or Honorarium: Medtronic, Comments: honorarium for Continuing Medical Education events. Tudor G. JovinRELATED: Support for Travel to Meetings for the Study or Other Purposes: Fundació Ictus; UNRELATED: Consultancy: Neuravi, Johnson \& Johnson; Employment: Stryker; Stock/Stock Options: FreeOx Biotech, Blockade Medical, Anaconda, Silk Road; Travel/Accommodations/Meeting Expenses Unrelated to Activities Listed: Stryker Neurovascular. Antoni Dávalos—RELATED: Grant: Covidien-Medtronic, Comments: unrestricted grant for conducting the REVASCAT trial*; UNRELATED: Payment for Lectures Including Service on Speakers Bureaus: Medtronic, Comments: less than US \$2000. Mayank Goyal—UNRELATED: Grant: Medtronic, Comments: part funding for the ESCAPE trial and funding for the HERMES collaboration*; Consulting Fee or Honorarium: Medtronic, Stryker, MicroVention, Comments: for education and advice related to acute stroke; Other: GE Healthcare, Comments: licensing agreement for systems of stroke diagnosis. *Money paid to the institution.

\section{REFERENCES}

1. Berkhemer OA, Fransen PS, Beumer D, et al. A randomized trial of intraarterial treatment for acute ischemic stroke. N Engl J Med 2015; 372:11-20 CrossRef Medline

2. Goyal M, Demchuk AM, Menon BK, et al; ESCAPE Trial Investigators. Randomized assessment of rapid endovascular treatment of ischemic stroke. N Engl J Med 2015;372:1019-30 CrossRef Medline

3. Campbell BC, Mitchell PJ, Kleinig TJ, et al; EXTEND-IA Investigators. Endovascular therapy for ischemic stroke with perfusion-imaging selection. $N$ Engl J Med 2015;372:1009-18 CrossRef Medline

4. Saver JL, Goyal M, Bonafe A, et al; SWIFT PRIME Investigators. Stent-retriever thrombectomy after intravenous t-PA vs. t-PA alone in stroke. $N$ Engl J Med 2015;372:2285-95 CrossRef Medline

5. Jovin TG, Chamorro A, Cobo E, et al; REVASCAT Trial Investigators. Thrombectomy within 8 hours after symptom onset in ischemic stroke. N Engl J Med 2015;372:2296-306 CrossRef Medline

6. Macrae IM. Preclinical stroke research: advantages and disadvantages of the most common rodent models of focal ischaemia. $\mathrm{Br} \mathrm{J}$ Pharmacol 2011;164:1062-78 CrossRef Medline

7. Duncan PW, Jorgensen HS, Wade DT. Outcome measures in acute stroke trials: a systematic review and some recommendations to improve practice. Stroke 2000;31:1429-38 CrossRef Medline

8. Quinn TJ, Dawson J, Walters MR, et al. Functional outcome measures in contemporary stroke trials. Int J Stroke 2009;4:200-05 Medline

9. Banks JL, Marotta CA. Outcomes validity and reliability of the modified Rankin scale: implications for stroke clinical trials: a literature review and synthesis. Stroke 2007;38:1091-96 CrossRef Medline

10. Thijs VN, Lansberg MG, Beaulieu C, et al. Is early ischemic lesion volume on diffusion-weighted imaging an independent predictor of stroke outcome? A multivariable analysis. Stroke 2000;31:2597602 CrossRef Medline

11. Wardlaw JM, Keir SL, Bastin ME, et al. Is diffusion imaging appearance an independent predictor of outcome after ischemic stroke? Neurology 2002;59:1381-87 CrossRef Medline

12. Saver JL, Johnston $\mathrm{KC}$, Homer $\mathrm{D}$, et al. Infarct volume as a surrogate or auxiliary outcome measure in ischemic stroke clinical trials: the RANTTAS investigators. Stroke 1999;30:293-98 CrossRef Medline

13. Kimura K, Sakamoto Y, Iguchi Y, et al, Serial changes in ischemic lesion volume and neurological recovery after t-PA therapy. $\mathrm{J} \mathrm{Neu}$ rol Sci 2011;304:35-39 CrossRef Medline

14. Rha JH, Saver JL. The impact of recanalization on ischemic stroke outcome: a meta-analysis. Stroke 2007;38:967-73 CrossRef Medline

15. Al-Ajlan FS, Goyal M, Demchuk AM, et al; ESCAPE Trial Investigators. Intra-arterial therapy and post-treatment infarct volumes: insights from the ESCAPE randomized controlled trial. Stroke 2016; 47:777-81 CrossRef Medline

16. Saver JL. Novel end point analytic techniques and interpreting shifts across the entire range of outcome scales in acute stroke trials. Stroke 2007;38:3055-62 CrossRef Medline

17. Kosior JC, Idris S, Dowlatshahi D, et al; PREDICT/Sunnybrook CTA ICH study investigators. Quantomo: validation of a computer-assisted methodology for the volumetric analysis of intracerebral haemorrhage. Int J Stroke 2011;6:302-05 CrossRef Medline

18. Zaidat OO, Yoo AJ, Khatri P, et al; STIR Thrombolysis in Cerebral Infarction (TICI) Task Force. Recommendations on angiographic revascularization grading standards for acute ischemic stroke: a consensus statement. Stroke 2013;44:2650-63 CrossRef Medline

19. Baron RM, Kenny DA. The moderator-mediator variable distinction in social psychological research: conceptual, strategic, and statistical considerations. J Pers Soc Psychol 1986;51:1173-82 CrossRef Medline

20. Sajobi TT, Menon BK, Wang M, et al; ESCAPE Trial Investigators. Early trajectory of stroke severity predicts long-term functional outcomes in ischemic stroke subjects: results from the ESCAPE Trial (Endovascular Treatment for Small Core and Anterior Circulation Proximal Occlusion With Emphasis on Minimizing CT to Recanalization Times). Stroke 2017;48:105-10 CrossRef Medline

21. Bulterijs S, Hull RS, Björk VC et al. It is time to classify biological aging as a disease. Front Genet 2015;6:205 CrossRef Medline 